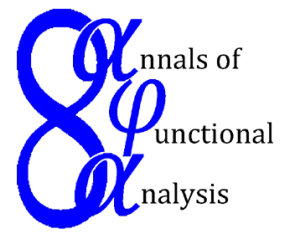

Ann. Funct. Anal. 7 (2016), no. 1, 170-179

http://dx.doi.org/10.1215/20088752-3428456

ISSN: 2008-8752 (electronic)

http://projecteuclid.org/afa

\title{
SOME CONSEQUENCES OF SPECTRAL SYNTHESIS IN HYPERGROUP ALGEBRAS
}

\begin{abstract}
B. E. FORREST
Dedicated to Professor Anthony To-Ming Lau, in recognition of his extraordinary contributions to our discipline and in great appreciation for all that he has done for the author and for so many others

Communicated by K. F. Taylor

Abstract. Properties of spectral synthesis are exploited to show that, for a large class of commutative hypergroups and for every compact hypergroup, every closed, reflexive, left-translation-invariant subspace of $L^{\infty}(K)$ is finitedimensional. Also, we show that, for a class of hypergroups which includes many commutative hypergroups and all $\mathcal{Z}$-hypergroups, every derivation of $L^{1}(K)$ into an arbitrary Banach $L^{1}$-bimodule is continuous.
\end{abstract}

\section{INTRODUCTION}

Spectral synthesis has been studied for hypergroups by various authors, including Chilana and Ross [2], Lasser [8], and Vogel [15], [16]. In this paper, we will consider two problems related to spectral synthesis of finite sets.

In Section 3, we show that if $K$ is either compact or if $K$ belongs to a rich class of commutative hypergroups, then every closed, reflexive, translation-invariant subspace of $L^{\infty}(K)$ must be finite-dimensional. This extends a beautiful theorem of Glicksberg [4] for compact groups and locally compact commutative groups.

In Section 4, we consider derivations of the hypergroup algebra $L^{1}(K)$. We prove that if $K$ is a $\mathcal{Z}$-hypergroup or if $K$ is in a subclass of those commutative hypergroups for which finite sets admit synthesis, then every derivation of $L^{1}(K)$

Copyright 2016 by the Tusi Mathematical Research Group.

Received Mar. 15, 2015; Accepted Aug. 11, 2015.

2010 Mathematics Subject Classification. Primary 43A62; Secondary 46H40.

Keywords. hypergroup algebras, spectral synthesis, Fourier algebra. 


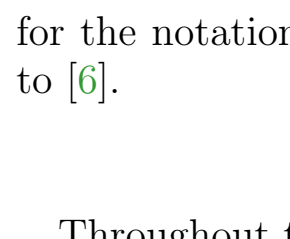

Ann. Funct. Anal. 7 (2016), no. 1, 170-179

http://dx.doi.org/10.1215/20088752-3428456

ISSN: 2008-8752 (electronic)

http://projecteuclid.org/afa

\title{
SOME CONSEQUENCES OF SPECTRAL SYNTHESIS IN HYPERGROUP ALGEBRAS
}

\begin{abstract}
B. E. FORREST
Dedicated to Professor Anthony To-Ming Lau, in recognition of his extraordinary contributions to our discipline and in great appreciation for all that he has done for the author and for so many others

Communicated by K. F. Taylor

Abstract. Properties of spectral synthesis are exploited to show that, for a large class of commutative hypergroups and for every compact hypergroup, every closed, reflexive, left-translation-invariant subspace of $L^{\infty}(K)$ is finitedimensional. Also, we show that, for a class of hypergroups which includes many commutative hypergroups and all $\mathcal{Z}$-hypergroups, every derivation of $L^{1}(K)$ into an arbitrary Banach $L^{1}$-bimodule is continuous.
\end{abstract}

\section{INTRODUCTION}

Spectral synthesis has been studied for hypergroups by various authors, including Chilana and Ross [2], Lasser [8], and Vogel [15], [16]. In this paper, we will consider two problems related to spectral synthesis of finite sets.

In Section 3, we show that if $K$ is either compact or if $K$ belongs to a rich class of commutative hypergroups, then every closed, reflexive, translation-invariant subspace of $L^{\infty}(K)$ must be finite-dimensional. This extends a beautiful theorem of Glicksberg [4] for compact groups and locally compact commutative groups.

In Section 4, we consider derivations of the hypergroup algebra $L^{1}(K)$. We prove that if $K$ is a $\mathcal{Z}$-hypergroup or if $K$ is in a subclass of those commutative hypergroups for which finite sets admit synthesis, then every derivation of $L^{1}(K)$

Copyright 2016 by the Tusi Mathematical Research Group.

Received Mar. 15, 2015; Accepted Aug. 11, 2015.

2010 Mathematics Subject Classification. Primary 43A62; Secondary 46H40.

Keywords. hypergroup algebras, spectral synthesis, Fourier algebra. 


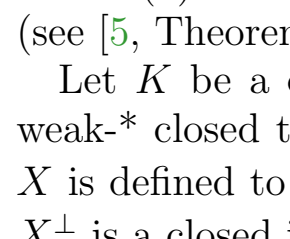

Ann. Funct. Anal. 7 (2016), no. 1, 170-179

http://dx.doi.org/10.1215/20088752-3428456

ISSN: 2008-8752 (electronic)

http://projecteuclid.org/afa

\title{
SOME CONSEQUENCES OF SPECTRAL SYNTHESIS IN HYPERGROUP ALGEBRAS
}

\begin{abstract}
B. E. FORREST
Dedicated to Professor Anthony To-Ming Lau, in recognition of his extraordinary contributions to our discipline and in great appreciation for all that he has done for the author and for so many others

Communicated by K. F. Taylor

Abstract. Properties of spectral synthesis are exploited to show that, for a large class of commutative hypergroups and for every compact hypergroup, every closed, reflexive, left-translation-invariant subspace of $L^{\infty}(K)$ is finitedimensional. Also, we show that, for a class of hypergroups which includes many commutative hypergroups and all $\mathcal{Z}$-hypergroups, every derivation of $L^{1}(K)$ into an arbitrary Banach $L^{1}$-bimodule is continuous.
\end{abstract}

\section{INTRODUCTION}

Spectral synthesis has been studied for hypergroups by various authors, including Chilana and Ross [2], Lasser [8], and Vogel [15], [16]. In this paper, we will consider two problems related to spectral synthesis of finite sets.

In Section 3, we show that if $K$ is either compact or if $K$ belongs to a rich class of commutative hypergroups, then every closed, reflexive, translation-invariant subspace of $L^{\infty}(K)$ must be finite-dimensional. This extends a beautiful theorem of Glicksberg [4] for compact groups and locally compact commutative groups.

In Section 4, we consider derivations of the hypergroup algebra $L^{1}(K)$. We prove that if $K$ is a $\mathcal{Z}$-hypergroup or if $K$ is in a subclass of those commutative hypergroups for which finite sets admit synthesis, then every derivation of $L^{1}(K)$

Copyright 2016 by the Tusi Mathematical Research Group.

Received Mar. 15, 2015; Accepted Aug. 11, 2015.

2010 Mathematics Subject Classification. Primary 43A62; Secondary 46H40.

Keywords. hypergroup algebras, spectral synthesis, Fourier algebra. 


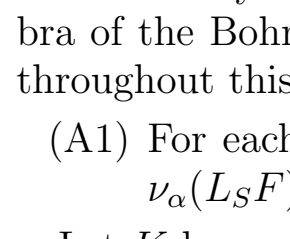

Ann. Funct. Anal. 7 (2016), no. 1, 170-179

http://dx.doi.org/10.1215/20088752-3428456

ISSN: 2008-8752 (electronic)

http://projecteuclid.org/afa

\title{
SOME CONSEQUENCES OF SPECTRAL SYNTHESIS IN HYPERGROUP ALGEBRAS
}

\begin{abstract}
B. E. FORREST
Dedicated to Professor Anthony To-Ming Lau, in recognition of his extraordinary contributions to our discipline and in great appreciation for all that he has done for the author and for so many others

Communicated by K. F. Taylor

Abstract. Properties of spectral synthesis are exploited to show that, for a large class of commutative hypergroups and for every compact hypergroup, every closed, reflexive, left-translation-invariant subspace of $L^{\infty}(K)$ is finitedimensional. Also, we show that, for a class of hypergroups which includes many commutative hypergroups and all $\mathcal{Z}$-hypergroups, every derivation of $L^{1}(K)$ into an arbitrary Banach $L^{1}$-bimodule is continuous.
\end{abstract}

\section{INTRODUCTION}

Spectral synthesis has been studied for hypergroups by various authors, including Chilana and Ross [2], Lasser [8], and Vogel [15], [16]. In this paper, we will consider two problems related to spectral synthesis of finite sets.

In Section 3, we show that if $K$ is either compact or if $K$ belongs to a rich class of commutative hypergroups, then every closed, reflexive, translation-invariant subspace of $L^{\infty}(K)$ must be finite-dimensional. This extends a beautiful theorem of Glicksberg [4] for compact groups and locally compact commutative groups.

In Section 4, we consider derivations of the hypergroup algebra $L^{1}(K)$. We prove that if $K$ is a $\mathcal{Z}$-hypergroup or if $K$ is in a subclass of those commutative hypergroups for which finite sets admit synthesis, then every derivation of $L^{1}(K)$

Copyright 2016 by the Tusi Mathematical Research Group.

Received Mar. 15, 2015; Accepted Aug. 11, 2015.

2010 Mathematics Subject Classification. Primary 43A62; Secondary 46H40.

Keywords. hypergroup algebras, spectral synthesis, Fourier algebra. 


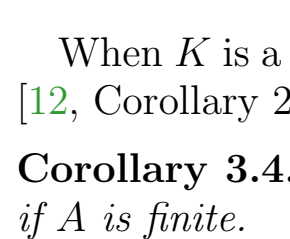

Ann. Funct. Anal. 7 (2016), no. 1, 170-179

http://dx.doi.org/10.1215/20088752-3428456

ISSN: 2008-8752 (electronic)

http://projecteuclid.org/afa

\title{
SOME CONSEQUENCES OF SPECTRAL SYNTHESIS IN HYPERGROUP ALGEBRAS
}

\begin{abstract}
B. E. FORREST
Dedicated to Professor Anthony To-Ming Lau, in recognition of his extraordinary contributions to our discipline and in great appreciation for all that he has done for the author and for so many others

Communicated by K. F. Taylor

Abstract. Properties of spectral synthesis are exploited to show that, for a large class of commutative hypergroups and for every compact hypergroup, every closed, reflexive, left-translation-invariant subspace of $L^{\infty}(K)$ is finitedimensional. Also, we show that, for a class of hypergroups which includes many commutative hypergroups and all $\mathcal{Z}$-hypergroups, every derivation of $L^{1}(K)$ into an arbitrary Banach $L^{1}$-bimodule is continuous.
\end{abstract}

\section{INTRODUCTION}

Spectral synthesis has been studied for hypergroups by various authors, including Chilana and Ross [2], Lasser [8], and Vogel [15], [16]. In this paper, we will consider two problems related to spectral synthesis of finite sets.

In Section 3, we show that if $K$ is either compact or if $K$ belongs to a rich class of commutative hypergroups, then every closed, reflexive, translation-invariant subspace of $L^{\infty}(K)$ must be finite-dimensional. This extends a beautiful theorem of Glicksberg [4] for compact groups and locally compact commutative groups.

In Section 4, we consider derivations of the hypergroup algebra $L^{1}(K)$. We prove that if $K$ is a $\mathcal{Z}$-hypergroup or if $K$ is in a subclass of those commutative hypergroups for which finite sets admit synthesis, then every derivation of $L^{1}(K)$

Copyright 2016 by the Tusi Mathematical Research Group.

Received Mar. 15, 2015; Accepted Aug. 11, 2015.

2010 Mathematics Subject Classification. Primary 43A62; Secondary 46H40.

Keywords. hypergroup algebras, spectral synthesis, Fourier algebra. 


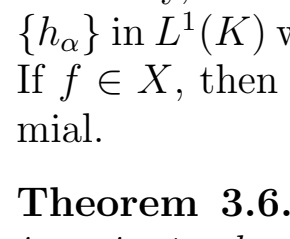

Ann. Funct. Anal. 7 (2016), no. 1, 170-179

http://dx.doi.org/10.1215/20088752-3428456

ISSN: 2008-8752 (electronic)

http://projecteuclid.org/afa

\title{
SOME CONSEQUENCES OF SPECTRAL SYNTHESIS IN HYPERGROUP ALGEBRAS
}

\begin{abstract}
B. E. FORREST
Dedicated to Professor Anthony To-Ming Lau, in recognition of his extraordinary contributions to our discipline and in great appreciation for all that he has done for the author and for so many others

Communicated by K. F. Taylor

Abstract. Properties of spectral synthesis are exploited to show that, for a large class of commutative hypergroups and for every compact hypergroup, every closed, reflexive, left-translation-invariant subspace of $L^{\infty}(K)$ is finitedimensional. Also, we show that, for a class of hypergroups which includes many commutative hypergroups and all $\mathcal{Z}$-hypergroups, every derivation of $L^{1}(K)$ into an arbitrary Banach $L^{1}$-bimodule is continuous.
\end{abstract}

\section{INTRODUCTION}

Spectral synthesis has been studied for hypergroups by various authors, including Chilana and Ross [2], Lasser [8], and Vogel [15], [16]. In this paper, we will consider two problems related to spectral synthesis of finite sets.

In Section 3, we show that if $K$ is either compact or if $K$ belongs to a rich class of commutative hypergroups, then every closed, reflexive, translation-invariant subspace of $L^{\infty}(K)$ must be finite-dimensional. This extends a beautiful theorem of Glicksberg [4] for compact groups and locally compact commutative groups.

In Section 4, we consider derivations of the hypergroup algebra $L^{1}(K)$. We prove that if $K$ is a $\mathcal{Z}$-hypergroup or if $K$ is in a subclass of those commutative hypergroups for which finite sets admit synthesis, then every derivation of $L^{1}(K)$

Copyright 2016 by the Tusi Mathematical Research Group.

Received Mar. 15, 2015; Accepted Aug. 11, 2015.

2010 Mathematics Subject Classification. Primary 43A62; Secondary 46H40.

Keywords. hypergroup algebras, spectral synthesis, Fourier algebra. 


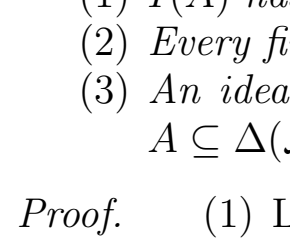

Ann. Funct. Anal. 7 (2016), no. 1, 170-179

http://dx.doi.org/10.1215/20088752-3428456

ISSN: 2008-8752 (electronic)

http://projecteuclid.org/afa

\title{
SOME CONSEQUENCES OF SPECTRAL SYNTHESIS IN HYPERGROUP ALGEBRAS
}

\begin{abstract}
B. E. FORREST
Dedicated to Professor Anthony To-Ming Lau, in recognition of his extraordinary contributions to our discipline and in great appreciation for all that he has done for the author and for so many others

Communicated by K. F. Taylor

Abstract. Properties of spectral synthesis are exploited to show that, for a large class of commutative hypergroups and for every compact hypergroup, every closed, reflexive, left-translation-invariant subspace of $L^{\infty}(K)$ is finitedimensional. Also, we show that, for a class of hypergroups which includes many commutative hypergroups and all $\mathcal{Z}$-hypergroups, every derivation of $L^{1}(K)$ into an arbitrary Banach $L^{1}$-bimodule is continuous.
\end{abstract}

\section{INTRODUCTION}

Spectral synthesis has been studied for hypergroups by various authors, including Chilana and Ross [2], Lasser [8], and Vogel [15], [16]. In this paper, we will consider two problems related to spectral synthesis of finite sets.

In Section 3, we show that if $K$ is either compact or if $K$ belongs to a rich class of commutative hypergroups, then every closed, reflexive, translation-invariant subspace of $L^{\infty}(K)$ must be finite-dimensional. This extends a beautiful theorem of Glicksberg [4] for compact groups and locally compact commutative groups.

In Section 4, we consider derivations of the hypergroup algebra $L^{1}(K)$. We prove that if $K$ is a $\mathcal{Z}$-hypergroup or if $K$ is in a subclass of those commutative hypergroups for which finite sets admit synthesis, then every derivation of $L^{1}(K)$

Copyright 2016 by the Tusi Mathematical Research Group.

Received Mar. 15, 2015; Accepted Aug. 11, 2015.

2010 Mathematics Subject Classification. Primary 43A62; Secondary 46H40.

Keywords. hypergroup algebras, spectral synthesis, Fourier algebra. 


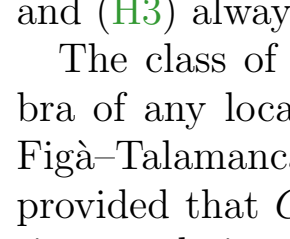

Ann. Funct. Anal. 7 (2016), no. 1, 170-179

http://dx.doi.org/10.1215/20088752-3428456

ISSN: 2008-8752 (electronic)

http://projecteuclid.org/afa

\title{
SOME CONSEQUENCES OF SPECTRAL SYNTHESIS IN HYPERGROUP ALGEBRAS
}

\begin{abstract}
B. E. FORREST
Dedicated to Professor Anthony To-Ming Lau, in recognition of his extraordinary contributions to our discipline and in great appreciation for all that he has done for the author and for so many others

Communicated by K. F. Taylor

Abstract. Properties of spectral synthesis are exploited to show that, for a large class of commutative hypergroups and for every compact hypergroup, every closed, reflexive, left-translation-invariant subspace of $L^{\infty}(K)$ is finitedimensional. Also, we show that, for a class of hypergroups which includes many commutative hypergroups and all $\mathcal{Z}$-hypergroups, every derivation of $L^{1}(K)$ into an arbitrary Banach $L^{1}$-bimodule is continuous.
\end{abstract}

\section{INTRODUCTION}

Spectral synthesis has been studied for hypergroups by various authors, including Chilana and Ross [2], Lasser [8], and Vogel [15], [16]. In this paper, we will consider two problems related to spectral synthesis of finite sets.

In Section 3, we show that if $K$ is either compact or if $K$ belongs to a rich class of commutative hypergroups, then every closed, reflexive, translation-invariant subspace of $L^{\infty}(K)$ must be finite-dimensional. This extends a beautiful theorem of Glicksberg [4] for compact groups and locally compact commutative groups.

In Section 4, we consider derivations of the hypergroup algebra $L^{1}(K)$. We prove that if $K$ is a $\mathcal{Z}$-hypergroup or if $K$ is in a subclass of those commutative hypergroups for which finite sets admit synthesis, then every derivation of $L^{1}(K)$

Copyright 2016 by the Tusi Mathematical Research Group.

Received Mar. 15, 2015; Accepted Aug. 11, 2015.

2010 Mathematics Subject Classification. Primary 43A62; Secondary 46H40.

Keywords. hypergroup algebras, spectral synthesis, Fourier algebra. 




Ann. Funct. Anal. 7 (2016), no. 1, 170-179

http://dx.doi.org/10.1215/20088752-3428456

ISSN: 2008-8752 (electronic)

http://projecteuclid.org/afa

\title{
SOME CONSEQUENCES OF SPECTRAL SYNTHESIS IN HYPERGROUP ALGEBRAS
}

\begin{abstract}
B. E. FORREST
Dedicated to Professor Anthony To-Ming Lau, in recognition of his extraordinary contributions to our discipline and in great appreciation for all that he has done for the author and for so many others

Communicated by K. F. Taylor

Abstract. Properties of spectral synthesis are exploited to show that, for a large class of commutative hypergroups and for every compact hypergroup, every closed, reflexive, left-translation-invariant subspace of $L^{\infty}(K)$ is finitedimensional. Also, we show that, for a class of hypergroups which includes many commutative hypergroups and all $\mathcal{Z}$-hypergroups, every derivation of $L^{1}(K)$ into an arbitrary Banach $L^{1}$-bimodule is continuous.
\end{abstract}

\section{INTRODUCTION}

Spectral synthesis has been studied for hypergroups by various authors, including Chilana and Ross [2], Lasser [8], and Vogel [15], [16]. In this paper, we will consider two problems related to spectral synthesis of finite sets.

In Section 3, we show that if $K$ is either compact or if $K$ belongs to a rich class of commutative hypergroups, then every closed, reflexive, translation-invariant subspace of $L^{\infty}(K)$ must be finite-dimensional. This extends a beautiful theorem of Glicksberg [4] for compact groups and locally compact commutative groups.

In Section 4, we consider derivations of the hypergroup algebra $L^{1}(K)$. We prove that if $K$ is a $\mathcal{Z}$-hypergroup or if $K$ is in a subclass of those commutative hypergroups for which finite sets admit synthesis, then every derivation of $L^{1}(K)$

Copyright 2016 by the Tusi Mathematical Research Group.

Received Mar. 15, 2015; Accepted Aug. 11, 2015.

2010 Mathematics Subject Classification. Primary 43A62; Secondary 46H40.

Keywords. hypergroup algebras, spectral synthesis, Fourier algebra. 




Ann. Funct. Anal. 7 (2016), no. 1, 170-179

http://dx.doi.org/10.1215/20088752-3428456

ISSN: 2008-8752 (electronic)

http://projecteuclid.org/afa

\title{
SOME CONSEQUENCES OF SPECTRAL SYNTHESIS IN HYPERGROUP ALGEBRAS
}

\begin{abstract}
B. E. FORREST
Dedicated to Professor Anthony To-Ming Lau, in recognition of his extraordinary contributions to our discipline and in great appreciation for all that he has done for the author and for so many others

Communicated by K. F. Taylor

Abstract. Properties of spectral synthesis are exploited to show that, for a large class of commutative hypergroups and for every compact hypergroup, every closed, reflexive, left-translation-invariant subspace of $L^{\infty}(K)$ is finitedimensional. Also, we show that, for a class of hypergroups which includes many commutative hypergroups and all $\mathcal{Z}$-hypergroups, every derivation of $L^{1}(K)$ into an arbitrary Banach $L^{1}$-bimodule is continuous.
\end{abstract}

\section{INTRODUCTION}

Spectral synthesis has been studied for hypergroups by various authors, including Chilana and Ross [2], Lasser [8], and Vogel [15], [16]. In this paper, we will consider two problems related to spectral synthesis of finite sets.

In Section 3, we show that if $K$ is either compact or if $K$ belongs to a rich class of commutative hypergroups, then every closed, reflexive, translation-invariant subspace of $L^{\infty}(K)$ must be finite-dimensional. This extends a beautiful theorem of Glicksberg [4] for compact groups and locally compact commutative groups.

In Section 4, we consider derivations of the hypergroup algebra $L^{1}(K)$. We prove that if $K$ is a $\mathcal{Z}$-hypergroup or if $K$ is in a subclass of those commutative hypergroups for which finite sets admit synthesis, then every derivation of $L^{1}(K)$

Copyright 2016 by the Tusi Mathematical Research Group.

Received Mar. 15, 2015; Accepted Aug. 11, 2015.

2010 Mathematics Subject Classification. Primary 43A62; Secondary 46H40.

Keywords. hypergroup algebras, spectral synthesis, Fourier algebra. 